\title{
Comparative Study of Ag Nanostructures: Molecular Simulations, Electrochemical Behavior, and Antibacterial Effect
}

\author{
Álvaro de Jesús Ruíz-Baltazar, ${ }^{1}$ Simón Yobanny Reyes-López, ${ }^{2}$ \\ D. Larrañaga, ${ }^{3}$ and R. Pérez ${ }^{3}$ \\ ${ }^{1}$ CONACyT-Centro de Física Aplicada y Tecnología Avanzada, Universidad Nacional Autónoma de México, \\ 76230 Juriquilla, QRO, Mexico \\ ${ }^{2}$ Instituto de Ciencias Biomédicas, Universidad Autónoma de Ciudad Juárez, Envolvente del PRONAF y Estocolmo s/n, \\ 32300 Ciudad Juárez, CHIH, Mexico \\ ${ }^{3}$ Centro de Física Aplicada y Tecnología Avanzada, Universidad Nacional Autónoma de México, \\ 76230 Juriquilla, QRO, Mexico
}

Correspondence should be addressed to Simón Yobanny Reyes-López; simon.reyes@uacj.mx

Received 8 September 2016; Accepted 25 October 2016

Academic Editor: Giuseppe Compagnini

Copyright ( 2016 Álvaro de Jesús Ruíz-Baltazar et al. This is an open access article distributed under the Creative Commons Attribution License, which permits unrestricted use, distribution, and reproduction in any medium, provided the original work is properly cited.

\begin{abstract}
Nanoparticles of Ag with different sizes and structures were obtained and studied. Two methods for reductions of Ag ions were employed, chemical reduction by sodium borohydride and ethylene glycol. Cuboctahedral and icosahedral structures were obtained. Molecular simulations were carried out in order to evaluate the reactivity of both structures. On the other hand, the electrochemical activity and antibacterial effect (E. coli) of the cuboctahedral and icosahedral structures were measured experimentally. The results obtained by molecular simulation, cyclic voltammetry, and antibacterial effect were compared and discussed in this work.
\end{abstract}

\section{Introduction}

Recently, the study of metallic nanoparticles has acquired particular interest due to the great number of potential applications on diverse science fields [1-3]. Specifically, silver nanoparticles have been widely studied due their particular physicochemical properties, including the notable electrical and thermal conductivity, a high catalytic activity, and surface-enhanced Raman scattering [4-6]. Consequently, AgNPs offer potential applications in chemistry, physics, biomedicine, catalysis, electronics, optics, environment engineering, and materials science [7-10]. Specifically, in biomedicine, AgNPs exhibit broad spectrum bactericidal and fungicidal activity [9]. The AgNPs have been synthesized by a great variety of chemical and physical methods. However, some reports indicate that the chemical reduction method provides well control under the structure and morphology of the AgNPs [11-15]. Specifically, reducing agents as sodium borohydride $\left(\mathrm{NaBH}_{4}\right)$ and ethylene glycol (EG) offer an excellent alternative to obtain silver nanoparticles sizes and morphologies controlled [16-22].

In this work, a comparative study regarding the nanostructure obtained as a function of the synthesis method is presented. On the other hand it has been reported that the structure observed for the Ag nanoparticles determines the properties and consequently their potential applications [23-26]. In this study, we focused on the behavior of the AgNPs obtained by the two synthesis methods proposed. Theoretical simulation using Density Functional Theory (DFT) was performed in order to calculate the values of the energy gap and binding energies associated with the AgNPs obtained. These values can be a great reference to determine the reactivity of the AgNPs. Additionally, cyclic voltammetry analysis and bactericidal tests were conducted. The theoretical and experimental data were compared and discussed widely. 


\section{Experimental Details}

\subsection{Synthesis of Ag Nanoparticles}

2.1.1. Polyol Method. Chemical synthesis of Ag nanoparticles was performed using ethylene glycol $\left(\mathrm{EG}, \mathrm{C}_{2} \mathrm{H}_{6} \mathrm{O}_{2}\right)$. Briefly, 3 vials with $9 \mathrm{~mL}$ of ethylene glycol (EG) were placed in an oil-bath of silicone. A polyvinylpirrolidone $1 \mathrm{M}$ (PVP, $\left.\left(\mathrm{C}_{6} \mathrm{H}_{9} \mathrm{NO}\right)_{n}\right)$ solution in EG was added. The mixture underwent magnetic stirring $(150 \mathrm{rpm})$ and was heated at $150^{\circ} \mathrm{C}$. Then three solutions of $\mathrm{AgNO}_{3}$ concentrations $0.2 \mathrm{M}, 0.1 \mathrm{M}$ and $0.05 \mathrm{M}$ were adhered to the solution of PVP and EG.

2.1.2. Chemical Reduction by Sodium Borohydride $\mathrm{NaBH}_{4}$. To reduce the $\mathrm{Ag}$ ions, aqueous solutions of $\mathrm{NaBH}_{4}(0.2 \mathrm{M})$ were added dropwise to the PVP containing metal ion mixtures. The chemical reduction process was carried out at room temperature. Ag concentrations of $0.05,0.1$, and $0.2 \mathrm{M}$ were prepared.

2.2. Structural Characterization. The structural and morphological characteristics of the dispersed metallic nanoparticles have been studied using a Philips Tecnai F20 transmission electron microscope with a field emission gun attachment and dot-to-dot direct maximum resolution of $0.23 \mathrm{~nm}$. TEM specimens were prepared by dispersing and subsequent drying of a drop of colloidal solution on a copper grid ( $3 \mathrm{~mm}$ in diameter) covered with an amorphous carbon film. Analysis of Fourier transform infrared (FT-IR) and Raman (RMN) spectroscopy were conducted. Additionally we employed the technique of UV-Vis spectroscopy as a rapid and reliable technique for characterization of silver nanoparticles, through a fiber optic spectrometer Ocean Optics model USB4F01962, in a range of 200 from $900 \mathrm{~nm}$ wavelength, with an integration time of 17 milliseconds and 10 scans.

2.3. Cyclic Voltammetry. The cyclic voltammetry of the AgNPs was carried out in acid medium employing a solution of $\mathrm{H}_{2} \mathrm{SO}_{4}(0.5 \mathrm{M})$, with scan rate $50 \mathrm{mVs}^{-1}$ at $25^{\circ} \mathrm{C}$ in the presence of $\mathrm{N}_{2}$. The AgNPs were supported on Vulcan carbon in order to measure the electrochemical activity.

2.4. Experimental Details of Antibacterial Effect. The antibacterial activity against Escherichia coli (E. coli) of silver nanoparticles synthesized with EG and $\mathrm{NaBH}_{4}$ as reducing agents was evaluated using the disk diffusion method. Three filter paper discs of $5 \mathrm{~mm}$ diameter were impregnated with $5 \mathrm{~mL}$ of AgNPs solutions at concentrations of 5, 10, and $15 \mathrm{mM}$. Subsequently, the discs were placed in the vial containing the E. coli. To evaluate the antibacterial activity of nanoparticles, a control disk was assessed too. The system was incubated at $36^{\circ} \mathrm{C}$ for $24 \mathrm{~h}$. Finally, the inhibition zone was measured.

\section{Results and Discussion}

3.1. Transmission Electron Microscopy (TEM). Figures 1(a) and 1(b) show low magnification TEM images of Ag nanoparticles produced by $\mathrm{NaBH}_{4}$ and $\mathrm{EG}$, respectively. In both cases, the morphology of the particles is semispherical and the formation of agglomerates is not appreciated. The histograms corresponding to the samples synthesized by $\mathrm{NaBH}_{4}$ and $\mathrm{EG}$ are insert in Figures 1(a) and 1(b), which indicates that the average particle sizes were $15 \mathrm{~nm}$ and $42 \mathrm{~nm}$, respectively. This fact can be attributed to the synthesis methodology and consequently to reduction rate of the reagent involved. The $\mathrm{NaBH}_{4}$ presents more capacity to reduce the Ag ions, because the reaction is carried out in 2 minutes, while in the case of the poliol method the reaction time is 2 hours. The Ag ions reduction by $\mathrm{NaBH}_{4}$ is carried out at room temperature. Figures 1(c) and 1(d) correspond to the high resolution transmission electron microscopy (HRTEM) images associated with $\mathrm{AgNPs}-\mathrm{NaBH}_{4}$ and AgNPs-EG, respectively. Specifically, in the case of $\mathrm{AgNPs}-\mathrm{NaBH}_{4}$, the $d$-spacing measured was $0.23 \mathrm{~nm}$ and $0.2 \mathrm{~nm}$ which corresponds, respectively, to the (111) and (200) planes of the FCC of the Ag structure. These values were obtained from the Fast Fourier Transform (FFT) and the HRTEM filtered images showed in the Figures 1(e) and 1(f). Regarding sample AgNPs-EG (Figure 1(d)), particles with multiple-twinned structures type [MTP] are observed. MTP particles were more commonly observed in the case of the samples produced by EG reducing agent. The particles obtained with ethylene glycol require the incorporation of heat for a period of $2 \mathrm{hr}$, which is carried out by nucleation of crystals of Ag. During this period formation of these crystalline defects is possible due to coalescence of nucleated fine particles. Therefore a decrease in reaction time can reduce particle sizes, crystalline defects, and well-defined morphologies. It is important to mention that the interplanar distances measured in this sample (AgNPs-EG) were $0.23 \mathrm{~nm}$ and 0.2 which belong to the (111) and (200) planes previously mentioned in figures (Figures $1(\mathrm{~g})$ and $1(\mathrm{~h})$ ). In order to discuss these results, it is important to note that the structure in both cases is different. The structure associated with AgNPs$\mathrm{NaBH}_{4}$ sample can be considered as a cubooctahedral (CO) structure, while the AgNPs-EG sample exhibits an icosahedral (Ih) structure. In this sense, the reactivity and, consequently, the electrochemical and antibacterial activity can be evaluated from the point of view of the structure observed.

\subsection{Spectroscopy Characterization}

3.2.1. Raman Spectroscopy. Figures 2(a) and 2(b) show the Raman spectroscopy of AgNPs-EG and AgNPs- $\mathrm{NaBH}_{4}$ samples, respectively. Regarding AgNPs-EG spectra, the bands located at 884,1050 , and $1090 \mathrm{~cm}^{-1}$ are attributed to the pure EG [27]. The intensity observed at $1276 \mathrm{~cm}^{-1}$ corresponds to the symmetric stretching mode of $\mathrm{C}-\mathrm{O}-\mathrm{C}$ present in the $\mathrm{EG}$. The band at $1458 \mathrm{~cm}^{-1}$ can be associated with the vibrations of the C-H group [10, 28, 29].

In the AgNPs- $\mathrm{NaBH}_{4}$ spectra, the band situated at $158 \mathrm{~cm}^{-1}$ shows clearly the presence of the Ag lattice vibrations models [30]. In these spectra can be appreciated the presence of the nitrate $\left(\mathrm{NO}^{-}\right)$functional group, which was dissociated in the aqueous medium employed during AgNPs$\mathrm{NaBH}_{4}$ synthesis. The intensities observed at 698, 717, and $791 \mathrm{~cm}^{-1}$ correspond to vibration of the doubly generated $\mathrm{N}$ $\mathrm{O}$ in plane bending $[31,32]$. Complementarily, the presence of 


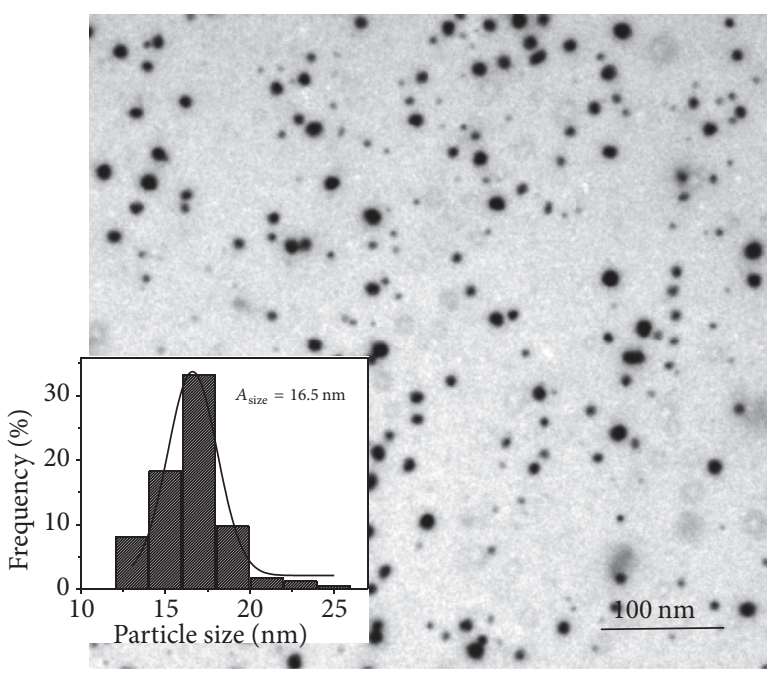

(a)

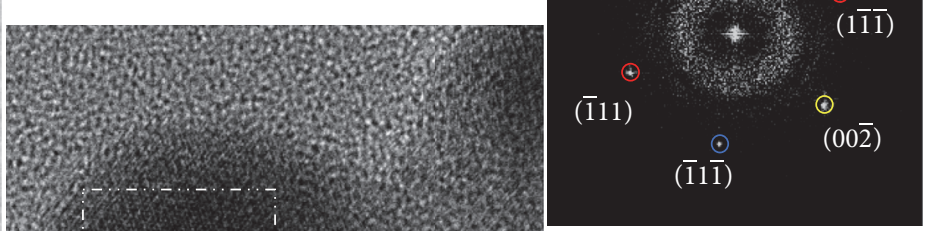

(e)

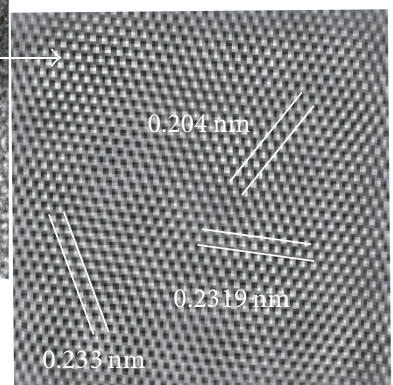

(f)

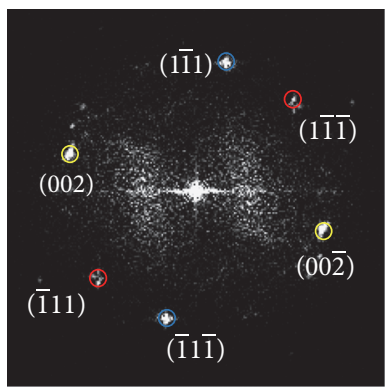

(g)

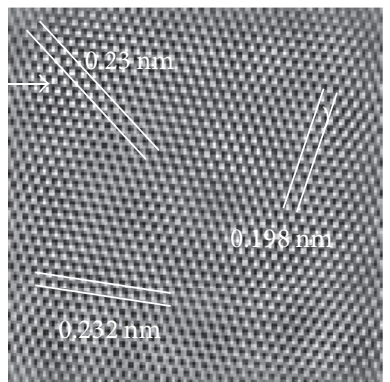

(h) (d)

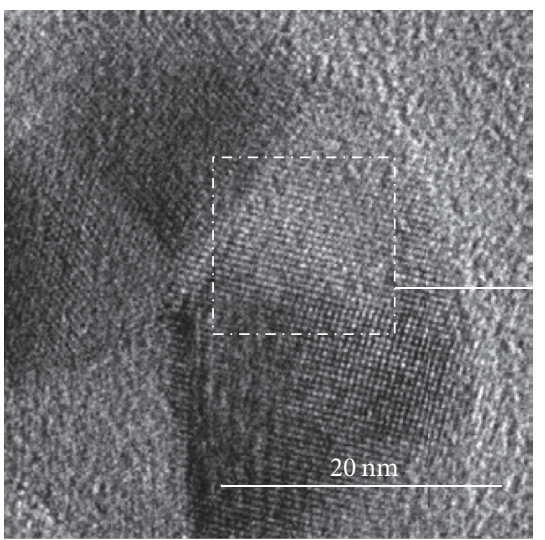

(b)

Figure 1: ((a) and (b)) Bright field TEM images corresponding to the AgNPs-NaBH 4 and AgNPs-EG samples. ((c) and (d)) HRTEM images of the AgNPs obtained by $\mathrm{NaBH}_{4}$ and EG. ((e) and (f)) Fast Fourier Transform (FFT) and HRTEM filtered image of AgNPs-NaBH . $_{4}$ ((g) and (h)) Fast Fourier Transform and HRTEM filtered image of AgNPs-EG.

the Ag nanoparticles is demonstrated by the bands situated at 1319 and $1643 \mathrm{~cm}^{-1}[33,34]$.

3.2.2. FT-IR Spectroscopy. Figure 2(c) shows the FT-IR spectra of the EG pure and the samples of AgNPs-EG at 5 and $10 \mathrm{mM}$. The bands observed at 1046 and $1424 \mathrm{~cm}^{-1}$ can be attributed to the vibrations bonds of the $\mathrm{C}-\mathrm{O}$ and $\mathrm{C}-\mathrm{O}-\mathrm{H}$, respectively $[6,35]$. The presence of the $\mathrm{NO}_{3}{ }^{-}$ion is confirmed by the band located at $1383 \mathrm{~cm}^{-1}$ [36]. The stretching vibrations of the hydroxyl functional $(\mathrm{OH})$ group can be associated with the bands located in the interval from 1600 to $2700 \mathrm{~cm}^{-1}$ [37]. However, the adsorption band situated at 


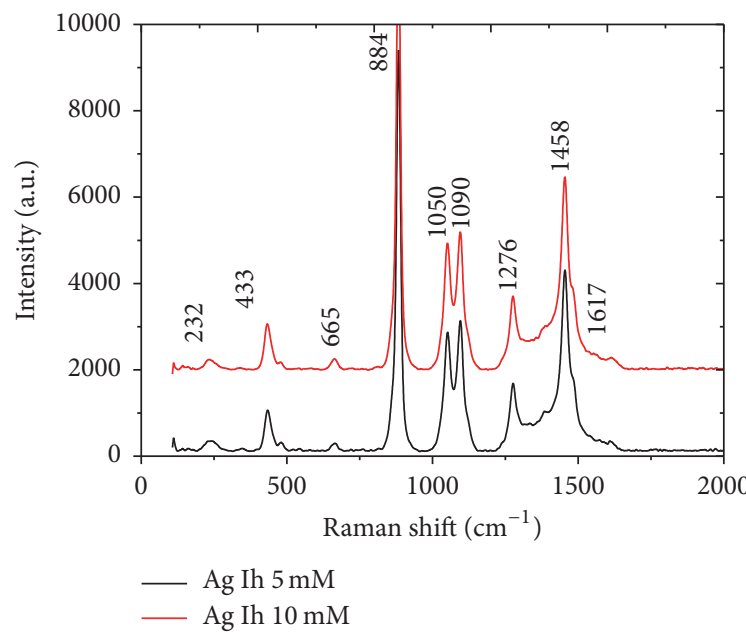

(a)

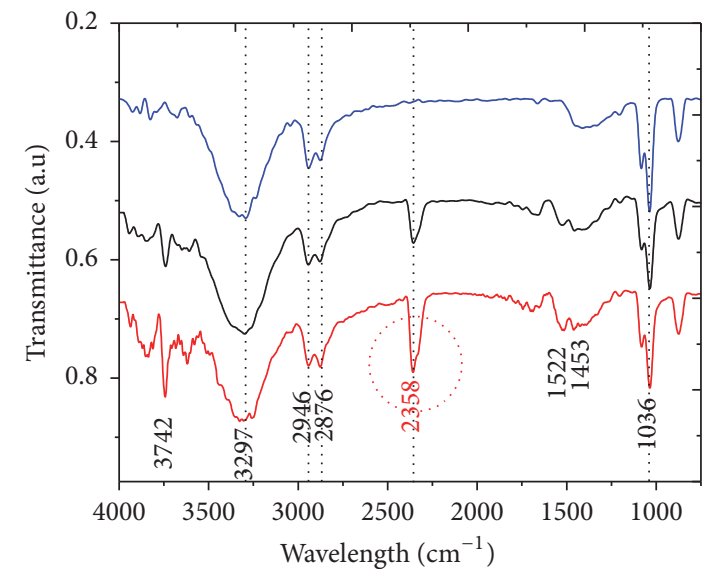

— Ethylene glycol _ $\quad$ Ag Ih $10 \mathrm{mM}$ — Ag Ih $5 \mathrm{mM}$

(c)

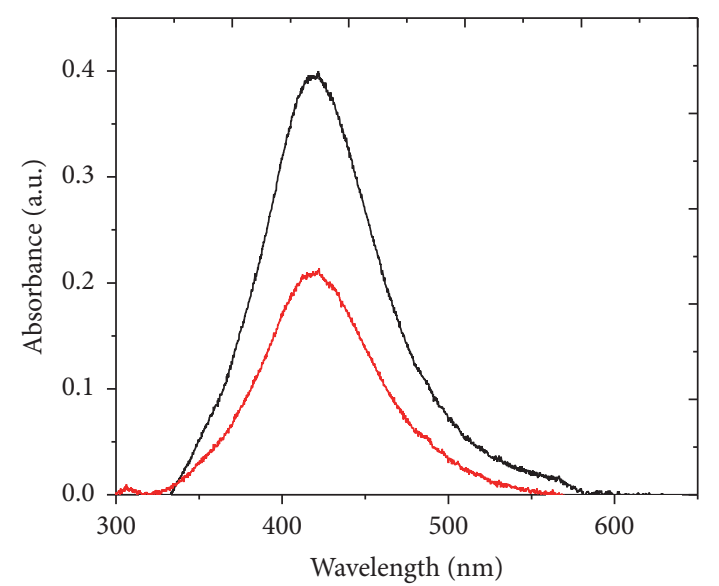

_ Ag Ih $10 \mathrm{mM}$
— Ag Ih $5 \mathrm{mM}$

(e)

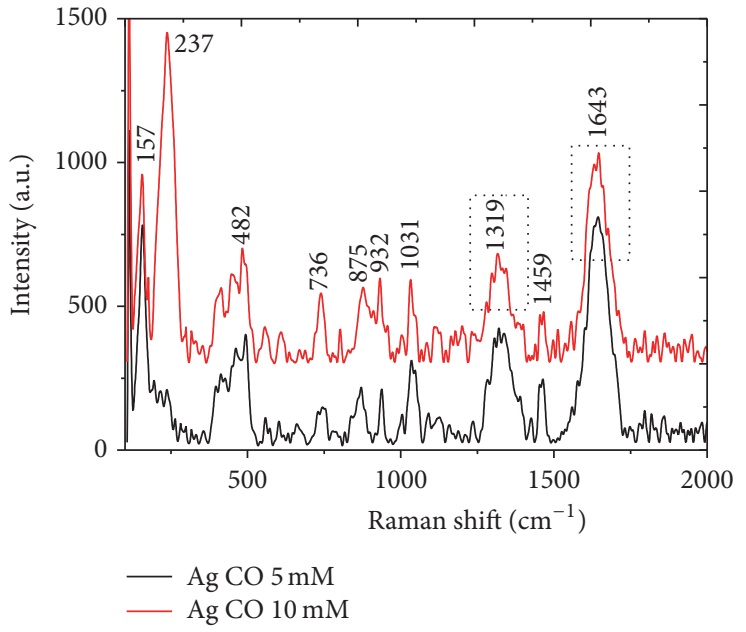

(b)

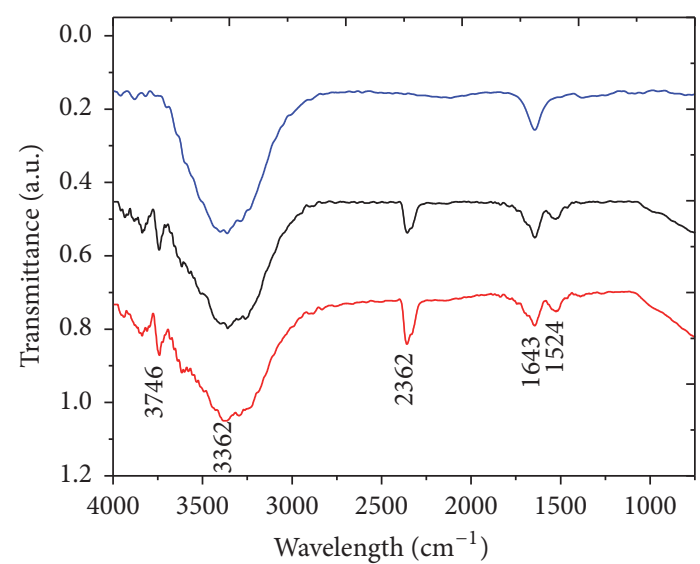

— Deionized water
Ag CO $5 \mathrm{mM}$

(d)

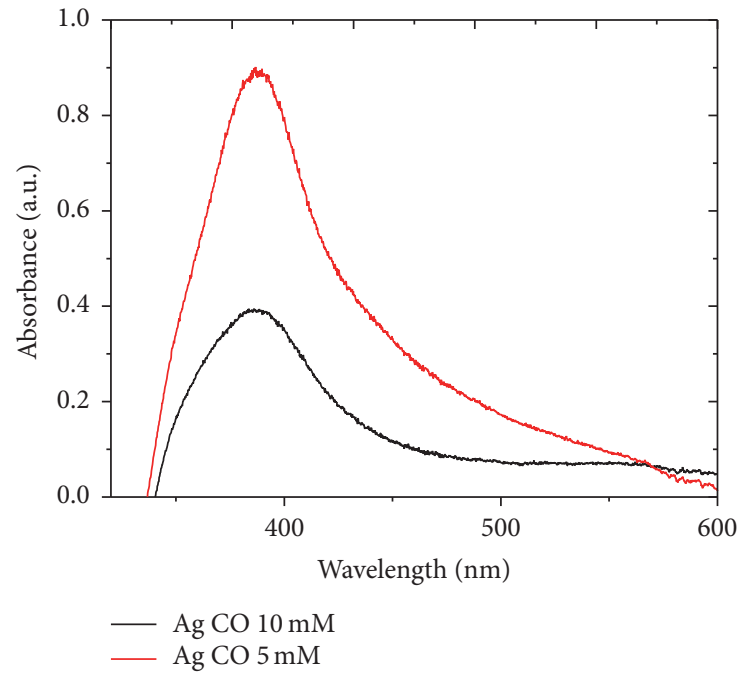

(f)

Figure 2: ((a) and (b)) Raman spectra of Ih and CO AgNPs. FT-IR spectra of AgNPs-EG (c) and AgNPs-NaBH 4 (d) samples. ((e) and (f)) UV-Vis analysis of Ih and CO Ag nanostructures, respectively. 
TABLE 1: Calculation of HOMO and LUMO energy levels of AgNPs.

\begin{tabular}{lccccc}
\hline Structure & HOMO $(\mathrm{Ha})$ & LUMO $(\mathrm{Ha})$ & Gap $(\mathrm{Ha})$ & Binding energy $(\mathrm{eV})$ & Binding energy/atom $(\mathrm{eV})$ \\
\hline Ag CO55 & -0.134055 & -0.119645 & 0.0144 & -98.38302 & -1.7888 \\
Ag Dh55 & -0.142562 & -0.11284 & 0.0297 & -99.03232 & -1.8006 \\
Ag Ih55 & -0.129181 & -0.096796 & 0.0324 & -100.82873 & -1.8332 \\
\hline
\end{tabular}

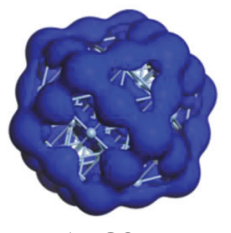

Ag CO55

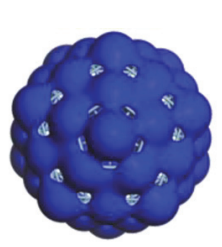

Ag Ih55

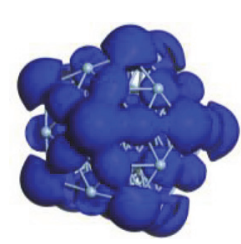

Ag Dh55

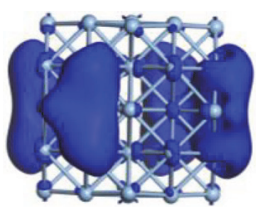

HOMO Ag CO55

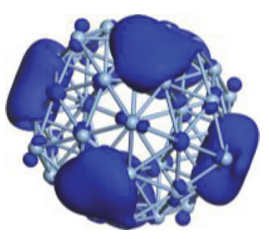

HOMO Ag Ih55

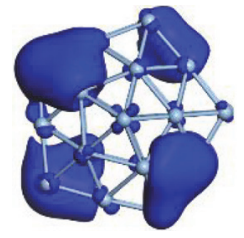

HOMO Ag Dh55

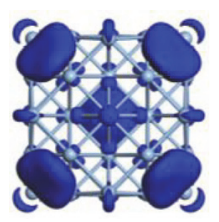

LUMO Ag CO55

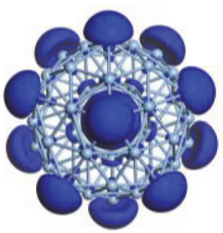

LUMO Ag Ih55

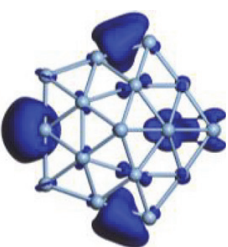

LUMO Dh55

FIGURE 3: Electrophilic fields of the Ag CO55 and Ag Dh55 models obtained at $0.0025 \mathrm{eV}$ isovalue distributions.

$2356 \mathrm{~cm}^{-1}$ confirms the presence of AgNPs [38]. In this case, this band is generated due to the interaction of the hydroxyl group of the EG and the partial charges of AgNPs [6, 38]. It is important to note that the peaks observed in the range of 1662 to $1587 \mathrm{~cm}^{-1}$ describe a maximum hydrogen bonding association, typical in the in the alcohol-water system [39]. Finally, the intensities at 2873 and $2930 \mathrm{~cm}^{-1}$ correspond to the stretching vibrations of the C-H bonds [36].

Figure 2(d) shows the infrared spectra of deionized water and the samples synthetized by $\mathrm{NaBH}_{4}(5 \mathrm{mM}$ and $10 \mathrm{mM})$. It is worth noting that the intensity at $2357 \mathrm{~cm}^{-1}$ is also observed in these FT-IR spectra. This fact confirms the AgNPs formation $[6,14,36,40]$. In this case, this band also is generated by the interaction of the hydroxyl group $\left(1646 \mathrm{~cm}^{-1}\right)$ of the deionized water and the partial charges of the AgNPs [40]. This result supports the TEM analysis.

3.2.3. UV-Vis Spectroscopy. In order to provide the full evidence with respect to the AgNPs presence, Figures 2(e) and 2(f) show the UV-Vis analysis corresponding to the CO and Ih samples, respectively. In these spectra, typical bands around $400 \mathrm{~nm}$ are observed in both cases [12, 41]. Specifically, for AgNPs-NaBH 4 and AgNPs-EG samples, bands located at 419 and $388 \mathrm{~nm}$ were observed, respectively. This result supports the particle size observed by TEM.

3.3. DFT Molecular Simulation. In order to describe the differences between the AgNP's configurations observed (cubooctahedral and icosahedral), DFT simulation of AgNP's models was carried out. Table 1 describes the HOMO and LUMO values calculated from the cubooctahedral, decahedral, and icosahedral configurations. These configurations were modeled with 55 atoms of Ag. Complementarily, the values of the energy gap and binding energies from the different AgNP's configurations were calculated. From the energy gap values, we can observe that the cubooctahedral structure (Ag CO55) exhibits the lowest energy HOMOLUMO gap $(0.0144 \mathrm{Ha})$, which is due to the higher density of electrons on its surface. With respect to the decahedral configurations, the energy gap is $0.0297 \mathrm{Ha}$. In this regard, it can expect theoretically a higher reactivity of the cubooctahedral configurations. Figure 3 shows an illustration of the electrophilic fields of the Ag CO55 and Ag Dh55 models obtained at $0.0025 \mathrm{eV}$ isovalue distributions.

It is clear that the electrophilic field of the cubooctahedron configuration is localized on the vortices, and for the icosahedral configuration the electrophilic field is localized 


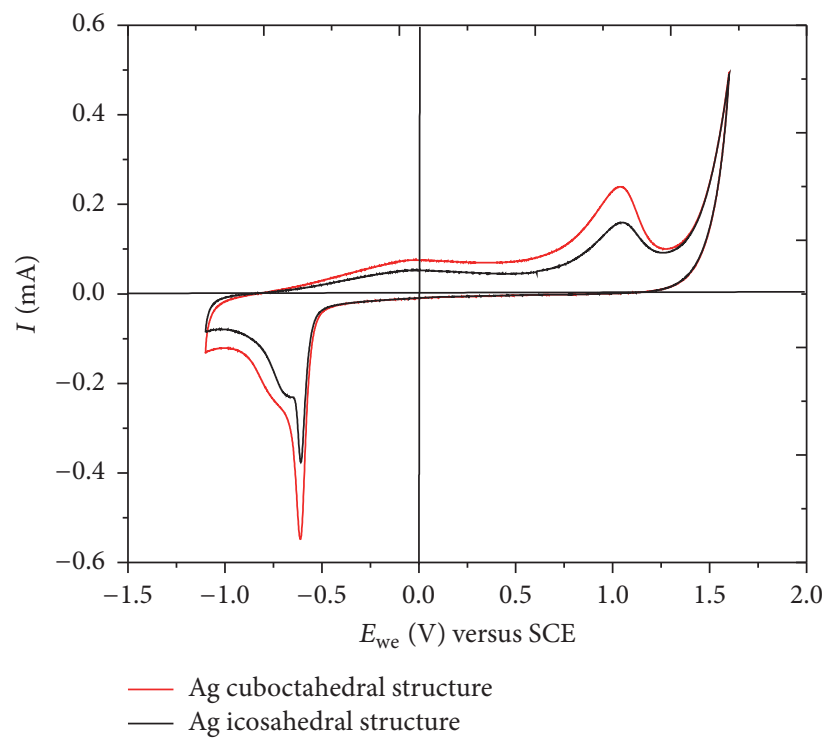

FIGURE 4: Cyclic voltammogram of the icosahedral and cuboctahedral structures of Ag nanoparticles in acid medium evaluated with a solution of $\mathrm{H}_{2} \mathrm{SO}_{4}(0.5 \mathrm{M})$. Scan rate $50 \mathrm{mVs}^{-1}$ at $25^{\circ} \mathrm{C}$ in the presence of $\mathrm{N}_{2}$.

on the face with fivefold symmetry. However, it is important to note that the size of the AgNPs model was the same in both cases. In this sense, it has been reported that the reactivity of the AgNPs can vary with respect to particle size; for example, only small decahedral structures exhibit superior catalytic activity compared with cubooctahedral structures [39, 40, 42-44]. In this case, the experimental results observed by TEM images indicate that, in AgNPs$\mathrm{NaBH}_{4}$ sample, the cubooctahedral structures appear with major frequency and with a significant minor particle size $(17 \mathrm{~nm})$ with respect to the AgNPs-EG sample $(40 \mathrm{~nm})$. For this reason a higher catalytic activity of AgNPs- $\mathrm{NaBH}_{4}$ sample would be expected. In this sense, the catalytic activity of the AgNPs- $\mathrm{NaBH}_{4}$ and AgNPs-EG structures can be evaluated by cyclic voltammetry.

3.4. Cyclic Voltammetry. In order to demonstrate experimentally the result obtained by DFT simulations, Figure 4 shows the cyclic voltammogram corresponding to the AgNPs with cubooctahedral (CO) and icosahedral (Ih) configuration. It can be seen that in the case of $\mathrm{CO}$ nanoparticles there is an increase in the current value of the redox processes involved in comparison with the Ih nanoparticles. Also, the cyclic voltammogram of both configurations is similar; no displacements are observed in the voltage values and they differ only in the associated current intensity reduction capability. This fact supports the theoretical analysis (DFT) presented previously.

3.5. Antibacterial Effect. The antibacterial activity of the AgNPs is showed in Figure 5. Figure 5(a) shows the antimicrobial property of AgNPs-EG associated with the decahedral structure. In this image can be appreciated the inhibition zone corresponding to AgNPs solution at concentrations of 5, 10, and $15 \mathrm{mM}$; the values measured in this case were $6.5,7.5$, and $10 \mathrm{~mm}$, respectively (Figure 5(c)). Regarding the AgNPs$\mathrm{NaBH}_{4}$ sample with cubooctahedral structure, Figure 5(b) illustrates in qualitative way the antibacterial effect with respect to the E. coli. Inhibitions zones of 7.5, 9 and $15 \mathrm{~mm}$ were observed in this case (Figure 5(c)). In this work, it was found that the growth inhibition effect in $S$. coli depends on the Ag concentration promoting good antimicrobial effect on lower Ag concentration (15 mM). These results confirmed the antibacterial properties of silver nanoparticles; however, the most significant one of these results is to compare the antibacterial effect as a function of the AgNPs structures. According to the theoretical (DFT analysis) and experimental (cyclic voltammetry) results obtained, the cubooctahedral structure $\left(\mathrm{AgNPs}-\mathrm{NaBH}_{4}\right)$ exhibits the highest catalytic and antibacterial activity.

\section{Conclusions}

The AgNPs synthesis route determines the structure obtained. Factors such as the reducing agent, the reduction rate, and temperature determine the formation of crystal defects in the Ag nanoparticles. The structure associated with the AgNPs- $\mathrm{NaBH}_{4}$ sample can be considered as a cubooctahedral structure, while the AgNPs-EG sample exhibits an icosahedral structure. Theoretical analyses (DFT) have made it possible to evaluate the reactivity of each structure and theoretically predict the reactivity of the AgNPs obtained. These results were proven experimentally by cyclic voltammetry and through a test of antibacterial effect. Due to their small size, the cubooctahedral structures obtained by $\mathrm{NaBH}_{4}$ were the most reactive and these AgNPs exhibit a major electrochemical and antibacterial activity. 


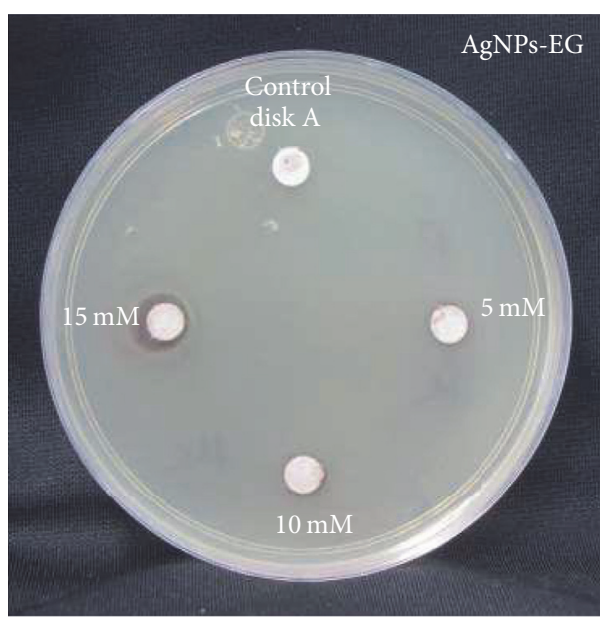

(a)

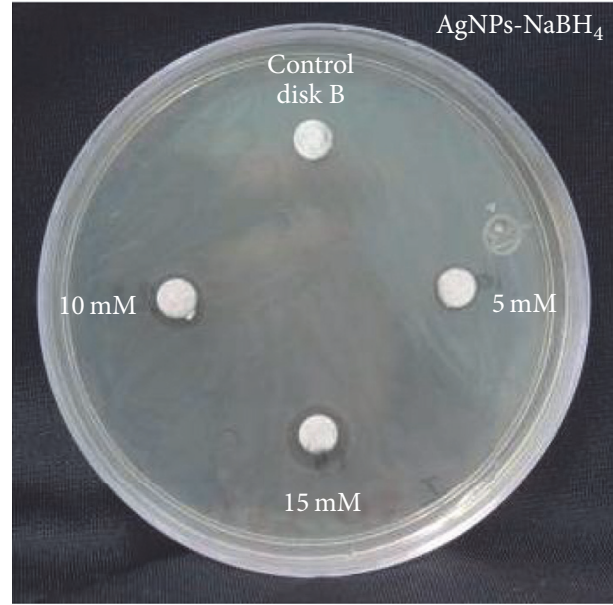

(b)

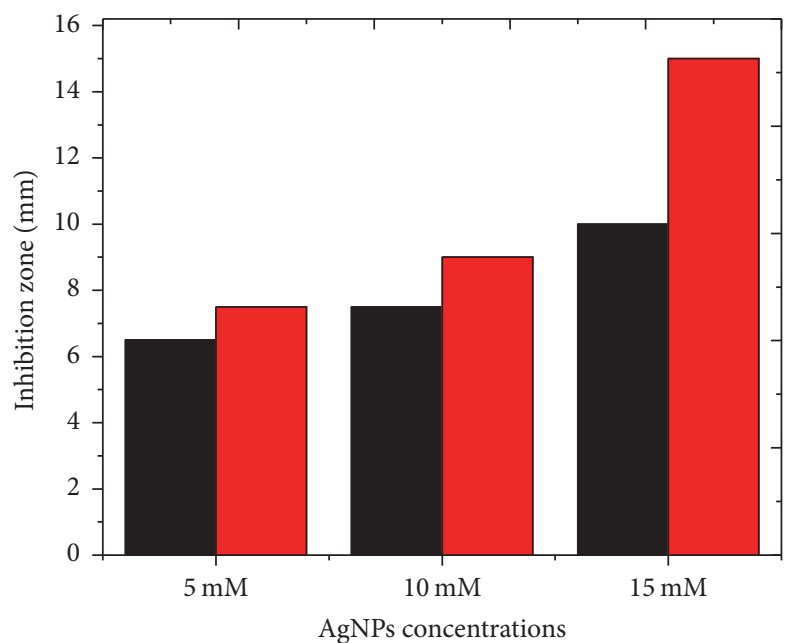

AgNPs-EG

AgNPs- $\mathrm{NaBH}_{4}$

(c)

Figure 5

\section{Competing Interests}

The authors declare no competing financial interests.

\section{Acknowledgments}

Álvaro de Jesús Ruíz-Baltazar appreciates the support provided by the Center of Applied Physics and Advanced Technology in collaboration with CONACyT through "Cátedras CONACyT" program.

\section{References}

[1] J. Yu and X. Zhou, "Synthesis of dendritic silver nanoparticles and their applications as SERS substrates," Advances in Materials Science and Engineering, vol. 2013, Article ID 519294, 4 pages, 2013.
[2] L. Yao, X. Song, G. Zhang et al., "Preparation of Ag/HBP/PAN nanofiber web and its antimicrobial and filtration property," Journal of Nanomaterials, vol. 2016, Article ID 4515769, 10 pages, 2016.

[3] M. A. San-Miguel, E. Z. da Silva, S. M. Zanetti et al., "In Situ growth of $\mathrm{Ag}$ nanoparticles on $\alpha-\mathrm{Ag}_{2} \mathrm{WO}_{4}$ under electron irradiation: probing the physical principles," Nanotechnology, vol. 27, no. 22, Article ID 225703, 2016.

[4] C. Pettegrew, Z. Dong, M. Z. Muhi, S. Pease, M. A. Mottaleb, and M. R. Islam, "Silver nanoparticle synthesis using monosaccharides and their growth inhibitory activity against gram-negative and positive bacteria," ISRN Nanotechnology, vol. 2014, Article ID 480284, pp. 1-8, 2014.

[5] S. Liang, G. Zhang, J. Min, J. Ding, and X. Jiang, "Synthesis and antibacterial testing of silver/poly (ether amide) composite nanofibers with ultralow silver content," Journal of Nanomaterials, vol. 2014, Article ID 684251, 10 pages, 2014. 
[6] A. Ruíz-Baltazar, R. Esparza, R. Pérez, and G. Rosas, "Spectroscopy study of silver nanoparticles produced by chemical reduction," Materials Science Forum, vol. 755, pp. 15-20, 2013.

[7] C. Li, J. Sun, D. Chen et al., “Ag-decorated $\mathrm{Fe}_{3} \mathrm{O}_{4} @ \mathrm{SiO}_{2}$ nanorods: synthesis, characterization, and applications in degradation of organic dyes," Journal of Nanomaterials, vol. 2016, Article ID 5837406, 8 pages, 2016.

[8] J. Lee, S. Shin, S. Kang, S. Lee, J. Seo, and T. Lee, "Highly stable surface-enhanced raman spectroscopy substrates using few-layer graphene on silver nanoparticles," Journal of Nanomaterials, vol. 2015, Article ID 975043, 7 pages, 2015.

[9] L. Körösi, M. Rodio, D. Dömötör et al., "Ultrasmall, ligand-free Ag nanoparticles with high antibacterial activity prepared by pulsed laser ablation in liquid," Journal of Chemistry, vol. 2016, Article ID 4143560, 8 pages, 2016.

[10] A. A. Semenova, A. P. Semenov, E. A. Gudilina et al., "Nanostructured silver materials for noninvasive medical diagnostics by surface-enhanced Raman spectroscopy," Mendeleev Communications, vol. 26, no. 3, pp. 177-186, 2016.

[11] Y. Sun, B. Mayers, T. Herricks, and Y. Xia, "Polyol synthesis of uniform silver nanowires: a plausible growth mechanism and the supporting evidence," Nano Letters, vol. 3, no. 7, pp. 955960, 2003.

[12] E. Fazio, S. Trusso, and R. C. C. Ponterio, "Surface-enhanced Raman scattering study of organic pigments using silver and gold nanoparticles prepared by pulsed laser ablation," Applied Surface Science, vol. 272, pp. 36-41, 2013.

[13] R. P. Chahal, S. Mahendia, A. K. Tomar, and S. Kumar, "UV irradiated PVA-Ag nanocomposites for optical applications," Applied Surface Science, vol. 343, pp. 160-165, 2015.

[14] W. Li, C. Sun, B. Hou, and X. Zhou, "Room temperature synthesis and catalytic properties of surfactant-modified Ag nanoparticles," International Journal of Spectroscopy, vol. 2012, Article ID 638692, 7 pages, 2012.

[15] J. Maley, G. Schatte, J. Yang, and R. Sammynaiken, "Spontaneous Ag-nanoparticle growth at single-walled carbon nanotube defect sites: a tool for in situ generation of SERS substrate," Journal of Nanotechnology, vol. 2011, Article ID 408151, 7 pages, 2011.

[16] K. Jia, H. Shou, P. Wang, X. Zhou, and X. Liu, "Controlled synthesis of silver nanostructures stabilized by fluorescent polyarylene ether nitrile," Applied Surface Science, vol. 377, pp. 180-183, 2016.

[17] A. I. Titkov, E. Y. Gerasimov, M. V. Shashkov et al., "Specific features of polyol synthesis of silver nanoparticles with the use of solid carboxylates as precursors," Colloid Journal, vol. 78, no. 4, pp. 515-524, 2016.

[18] J. Helmlinger, O. Prymak, K. Loza, M. Gocyla, M. Heggen, and M. Epple, "On the crystallography of silver nanoparticles with different shapes," Crystal Growth \& Design, vol. 16, no. 7, pp. 3677-3687, 2016.

[19] D. R. Whitcomb, A. R. Clapp, P. Bühlmann, J. C. Blinn, and J. Zhang, "New perspectives on silver nanowire formation from dynamic silver ion concentration monitoring and nitric oxide production in the polyol process," Crystal Growth \& Design, vol. 16, no. 4, pp. 1861-1868, 2016.

[20] M. K. Temgire and S. S. Joshi, "Optical and structural studies of silver nanoparticles," Radiation Physics and Chemistry, vol. 71, no. 5, pp. 1039-1044, 2004.

[21] L.-C. Chen, D.-C. Tien, N. Van Thai, and S. Ashraf, "Study of Ag and Au nanoparticles synthesized by Arc discharge in deionized water," Journal of Nanomaterials, vol. 2010, Article ID 634757, 9 pages, 2010.

[22] M. Ghanipour and D. Dorranian, "Effect of Ag-nanoparticles doped in polyvinyl alcohol on the structural and optical properties of PVA films," Journal of Nanomaterials, vol. 2013, Article ID 897043, 10 pages, 2013.

[23] B. Lim, Y. Xiong, and Y. Xia, "A water-based synthesis of octahedral, decahedral, and icosahedral Pd nanocrystals," Angewandte Chemie-International Edition, vol. 46, no. 48, pp. 9279-9282, 2007.

[24] D. Seo, C. I. Yoo, I. S. Chung, S. M. Park, S. Ryu, and H. Song, "Shape adjustment between multiply twinned and singlecrystalline polyhedral gold nanocrystals: decahedra, icosahedra, and truncated tetrahedra," The Journal of Physical Chemistry C, vol. 112, no. 7, pp. 2469-2475, 2008.

[25] M. Tsuji, M. Ogino, R. Matsuo et al., "Stepwise growth of decahedral and icosahedral silver nanocrystals in DMF," Crystal Growth and Design, vol. 10, no. 1, pp. 296-301, 2010.

[26] Q. Zhang, J. Xie, J. Yang, and J. Y. Lee, "ARTICLE and superlattice formation," Society, vol. 3, no. 1, pp. 139-148, 2009.

[27] L.-F. Zhang and C.-Y. Zhang, "Dodecahedral Au@Pd nanocrystals with high-index facets and excellent electrocatalytic activity and highly efficient surface-enhanced Raman scattering enhancement," Nanoscale, vol. 5, no. 13, pp. 6074-6080, 2013.

[28] L. Rosa, M. Ranjan, M. Bhatnagar, D. Mortazavi, S. Mukherjee, and S. Juodkazis, "Simulation and measurement of solar harvesting enhancement of silver plasmonic nanoparticles on GaSb nanodots," Journal of Photonics, vol. 2014, Article ID 327586, 7 pages, 2014.

[29] H. Song, X. Li, S. Yoo et al., "Highly sensitive surface enhanced raman spectroscopy from $\mathrm{Ag}$ nanoparticles decorated graphene sheet," Journal of Nanomaterials, vol. 2014, Article ID 538024, 7 pages, 2014.

[30] L. Zhang, B. Wang, G. Zhu, and X. Zhou, "Synthesis of silver nanowires as a SERS substrate for the detection of pesticide thiram," Spectrochimica Acta Part A: Molecular and Biomolecular Spectroscopy, vol. 133, pp. 411-416, 2014.

[31] J. Jiang, O.-Y. Lei, L. Zhu, J. Zou, and H. Tang, "Novel one-pot fabrication of lab-on-a-bubble@Ag substrate without coupling-agent for surface enhanced Raman scattering," Scientific Reports, vol. 4, article no. 3942, 2014.

[32] C. Lofrumento, F. Arci, S. Carlesi, M. Ricci, E. Castellucci, and M. Becucci, "Safranin-O dye in the ground state. A study by density functional theory, Raman, SERS and infrared spectroscopy," Spectrochimica Acta Part A: Molecular and Biomolecular Spectroscopy, vol. 137, pp. 677-684, 2015.

[33] I. Martina, R. Wiesinger, D. Jembrih-Simbürger, and $M$. Schreiner, "Micro-Raman characterisation of silver corrosion products: instrumental set up and reference database," $e$ Preservation Science, vol. 9, pp. 1-8, 2012.

[34] T. B. Nguyen, T. K. Thu Vu, Q. D. Nguyen, T. D. Nguyen, T. A. Nguyen, and T. H. Trinh, "Preparation of metal nanoparticles for surface enhanced Raman scattering by laser ablation method," Advances in Natural Sciences: Nanoscience and Nanotechnology, vol. 3, no. 2, Article ID 025016, 2012.

[35] L. Ouyang, L. Zhu, J. Jiang, and H. Tang, "A surface-enhanced Raman scattering method for detection of trace glutathione on the basis of immobilized silver nanoparticles and crystal violet probe," Analytica Chimica Acta, vol. 816, pp. 41-49, 2014.

[36] A. Ungurean, M. Oltean, L. David, N. Leopold, J. P. Prates Ramalho, and V. Chiş, "Adsorption of sulfamethoxazole 
molecule on silver colloids: a joint SERS and DFT study," Journal of Molecular Structure, vol. 1073, pp. 71-76, 2014.

[37] C. A. Smyth, S. Mehigan, Y. P. Rakovich, S. E. J. Bell, and E. M. McCabe, "Pterin detection using surface-enhanced Raman spectroscopy incorporating a straightforward silver colloidbased synthesis technique," Journal of Biomedical Optics, vol. 16, no. 7, Article ID 077007, 2011.

[38] L. Ramanauskaite and V. Snitka, "The synthesis of controlled shape nanoplasmonic silver-silica structures by combining solgel technique and direct silver reduction," Nanoscale Research Letters, vol. 10, no. 1, p. 133, 2015.

[39] V. Uzayisenga, X.-D. Lin, L.-M. Li et al., "Synthesis, characterization, and 3D-FDTD simulation of $\mathrm{Ag@SiO} 2$ nanoparticles for shell-isolated nanoparticle-enhanced raman spectroscopy," Langmuir, vol. 28, no. 24, pp. 9140-9146, 2012.

[40] Z. Xu and G. Hu, "Simple and green synthesis of monodisperse silver nanoparticles and surface-enhanced Raman scattering activity," RSC Advances, vol. 2, no. 30, pp. 11404-11409, 2012.

[41] M. A. Ahmed, N. Hasan, and S. Mohiuddin, "Silver nanoparticles: green synthesis, characterization, and their usage in determination of mercury contamination in seafoods," ISRN Nanotechnology, vol. 2014, Article ID 148184, 5 pages, 2014.

[42] C. Du, B. Wang, F. Sun et al., "Refractive index sensitivities of plane Ag nanosphere cluster sensors," Sensors and Actuators B: Chemical, vol. 215, pp. 142-145, 2015.

[43] C. Jing and Y. Fang, "Experimental (SERS) and theoretical (DFT) studies on the adsorption behaviors of 1-cysteine on gold/silver nanoparticles," Chemical Physics, vol. 332, no. 1, pp. 27-32, 2007.

[44] K. Sivashanmugan, J.-D. Liao, P.-L. Shao, B. Haochih Liu, T.-Y. Tseng, and C.-Y. Chang, "Intense Raman scattering on hybrid $\mathrm{Au} / \mathrm{Ag}$ nanoplatforms for the distinction of MMP-9-digested collagen type-I fiber detection," Biosensors and Bioelectronics, vol. 72, pp. 61-70, 2015. 

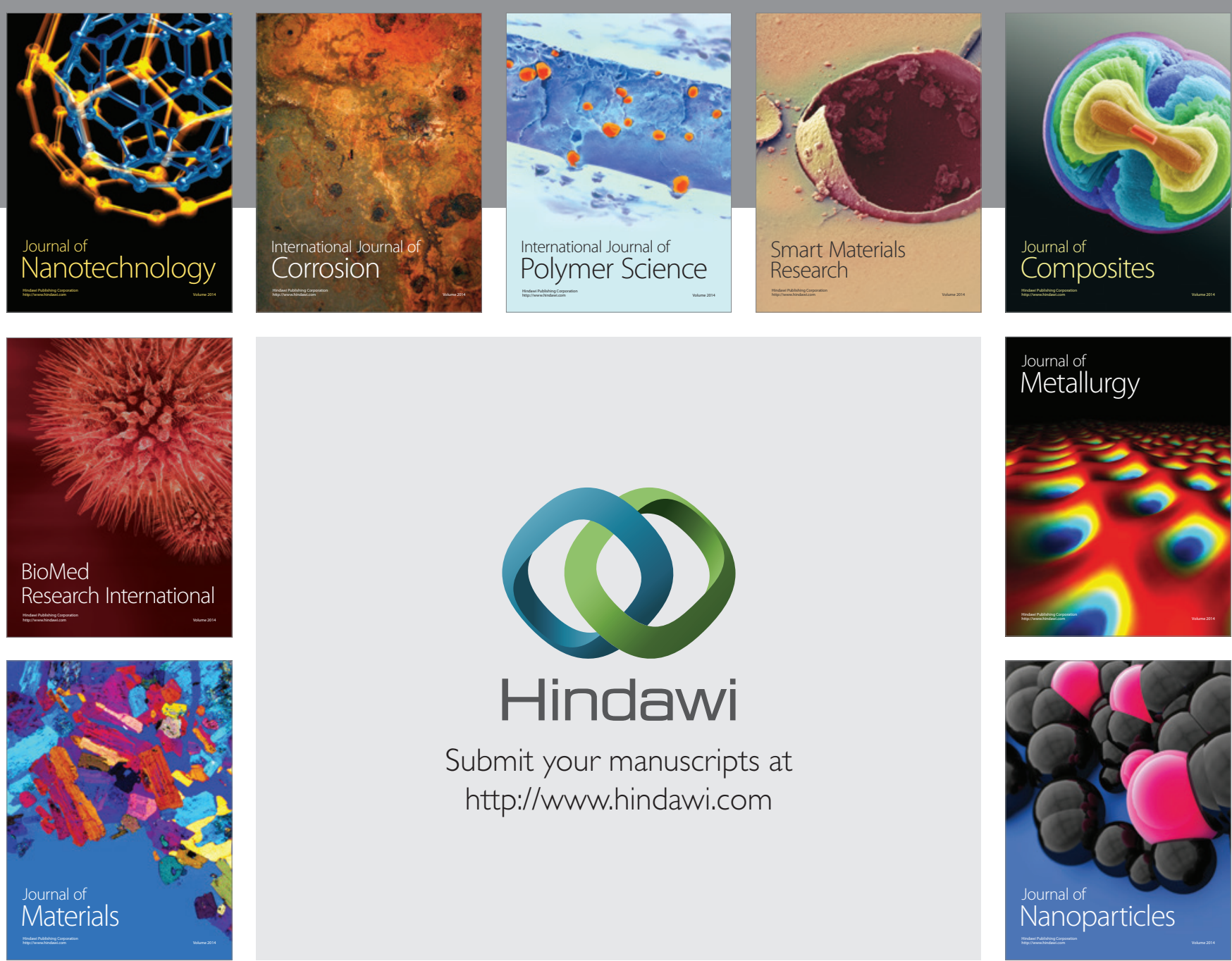

\section{Hindawi}

Submit your manuscripts at

http://www.hindawi.com

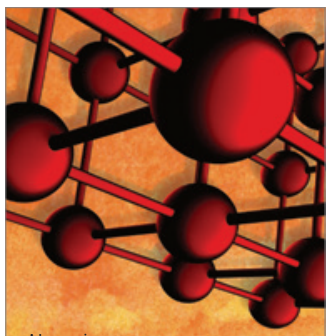

Materials Science and Engineering
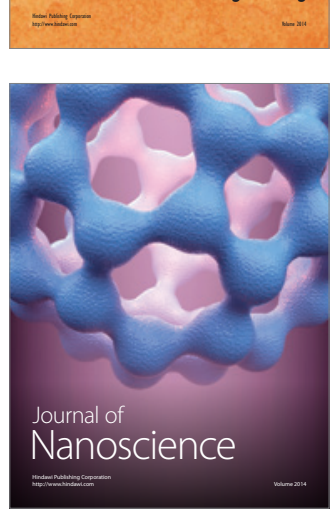
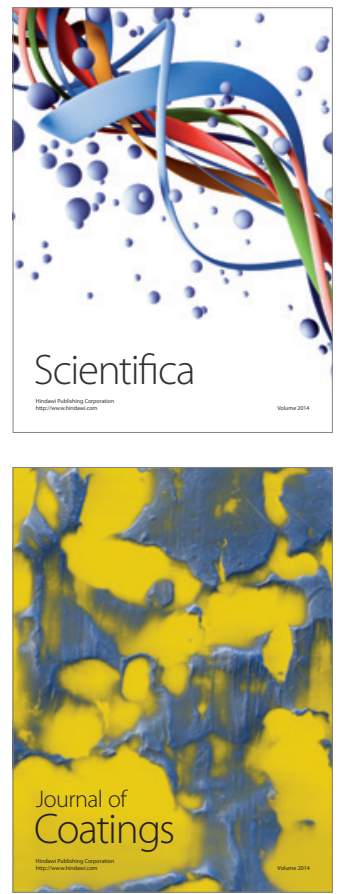
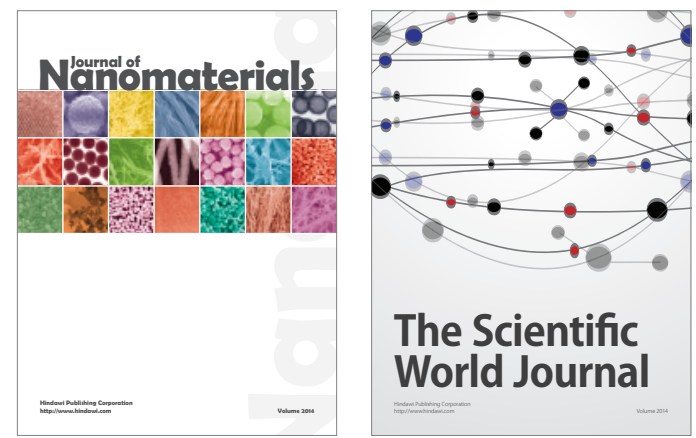

The Scientific World Journal
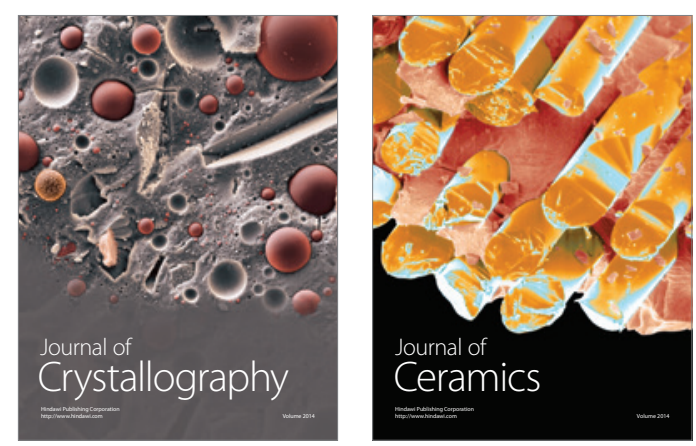
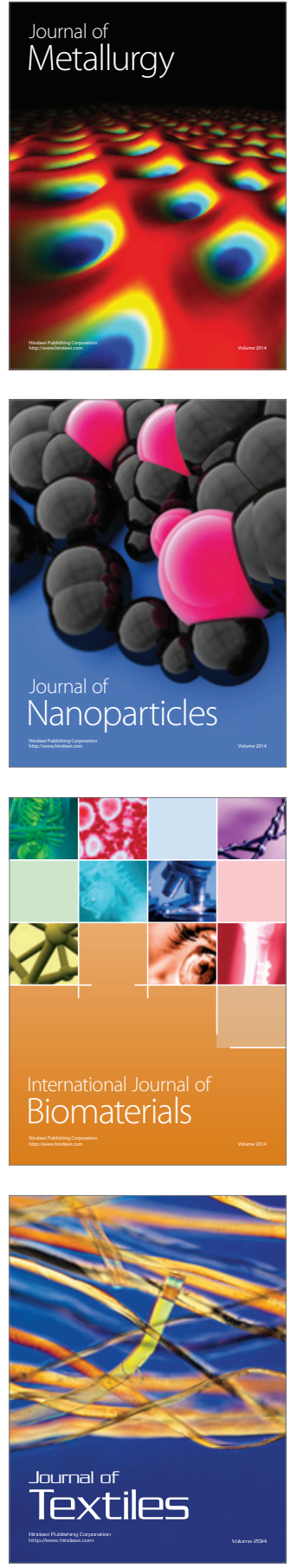